\title{
Transcriptional Waves Induced by ATP and LPS in Primary Microglial Culture Reveal Multiple Inflammatory States
}

Michal Linial ( $\square$ michall@cc.huji.ac.il)

The Hebrew University of Jerusalem

Keren Zohar

The Hebrew University of Jerusalem

Elyad Lezmi

The Hebrew University of Jerusalem

Fanny Reichert

The Hebrew University of Jerusalem

Tsiona Eliyahu

The Hebrew University of Jerusalem

Shlomo Rotshenker

The Hebrew University of Jerusalem

Marta Weinstock

The Hebrew University of Jerusalem

\section{Research Article}

Keywords: Innate immune system, IL-1, RNA-seq, Purinergic receptor, Inflammation, Cytokines

Posted Date: February 28th, 2022

DOI: https://doi.org/10.21203/rs.3.rs-1359348/v1

License: (c) (i) This work is licensed under a Creative Commons Attribution 4.0 International License.

Read Full License 


\section{Transcriptional Waves Induced by ATP and LPS in Primary Microglial Culture Reveal Multiple Inflammatory States}

Keren Zohar $^{1^{\dagger}}$, Elyad Lezmi ${ }^{2 \dagger}$, Fanny Reichert ${ }^{3}$, Tsiona Eliyahu ${ }^{1}$, Shlomo Rotshenker ${ }^{3}$, Marta Weinstock ${ }^{4}$ and Michal Linial ${ }^{1 *}$

${ }^{1}$ Department of Biological Chemistry, Institute of Life Sciences, The Hebrew University of Jerusalem

${ }^{2}$ Department of Genetics, Institute of Life Sciences, The Hebrew University of Jerusalem

${ }^{3}$ Department of Medical Neurobiology, IMRIC, Faculty of Medicine, The Hebrew University of Jerusalem

${ }^{4}$ Institute of Drug Research, School of Pharmacy, The Hebrew University of Jerusalem, Jerusalem, Israel

${ }^{+}$Equal contribution

*Correspondence: Corresponding author Michal Linial, Dept. of Biological Chemistry, Institute of Life Sciences, The Hebrew University of Jerusalem, Jerusalem, 91904, Israel; Phone: +972-54-8820035;

Email: michall@cc.huji.ac.il; Skype: michal_linial

Running title: Transcriptomics of activated primary microglia

Statistics:

Tables: 1

Figures: 8

References: 62

Word counts: Intro to Discussion (no Methods, Legends): 4415

Supplementary

Tables: Tables S1-S5

Figures: Figs. S1-S7 


\begin{abstract}
The primary role of microglia is to respond effectively to various disturbances to maintain brain homeostasis. In this study, we stimulated murine neonatal microglial cells with benzoyl ATP (bzATP) and lipopolysaccharide (LPS) and monitored their ability to secrete pro-inflammatory cytokines together with the changes in the transcriptome of the culture. Activation by bzATP, an agonist of purinergic receptors, induces a short-lived wave of transcriptional changes. However, a long-lasting transcriptional profile affecting thousands of genes occurs already at 3 hours after activation with a combination of bzATP and LPS. The transcriptomic profile is dominated by induced cytokines (e.g., IL1$\alpha$ and IL1- $\beta$ ) and chemokines, in addition to upregulation of cytokine membrane receptors and their direct regulators. We identified the TNF and NF- $\mathrm{KB}$ signaling pathways as the principal hubs for many differentially expressed genes. Many of the reported inflammatory-related genes were upregulated by orders of magnitude. We conclude that the kinetics of microglial activation and specific combinations of stimuli yield coordinated and robust transcriptional waves. We propose that the primary microglia culture could be beneficial for studying brain inflammation associated with aging, injury, and neurodegenerative diseases.
\end{abstract}

Keywords: Innate immune system; IL-1; RNA-seq; Purinergic receptor; Inflammation; Cytokines 


\section{Introduction}

Microglia act as the resident macrophages of the central nervous system (CNS). Their function is to maintain brain homeostasis and respond effectively to a broad spectrum of perturbations induced by acute stress from toxic agents or physical injury [1-3]. This is accomplished through communication with the surrounding neurons and astrocytes, and the release of signaling molecules like ATP (adenosine triphosphate), that interact with specific purinergic receptors on the microglial membrane $[4,5]$. To protect neurons from apoptotic death and remove dying cells, microglia respond by changing their mode of activation, and morphology [6-8]. During chronic stress and neuronal damage that occurs in aging, the release of pro-inflammatory cytokines becomes prolonged and together with oxidative stress, exacerbates neurodegeneration [9]. Glial cells with excess amounts of neuroinflammatory markers such as HLA-DR, CD68, and CD105 are abundant in Parkinson's (PD), Alzheimer's diseases (AD), and frontotemporal dementia [10].

To better understand the processes involved in the regulation of microglial activity, studies have been performed under controlled conditions in isolated microglia, prepared from neonatal rodents and cultured alone or with astrocytes [11]. Exposure of these cells to activators like lipopolysaccharide (LPS), interferon-gamma (IFN $\gamma$ ), interleukins, and other extracellular signaling molecules produces a change in their function, morphology, and inflammatory responses [12, 13]. LPS increases the release of TNF- $\alpha$ and other pro-inflammatory cytokines.

Cumulative evidence suggests that microglial inflammatory activity plays a role in age-dependent memory decline [14], synaptic plasticity [15, 16], neuroprotection [17], and neurodegeneration [18]. Moreover, it was shown that extracellular ATP triggers TNF- $\alpha$ release through purinergic P2 receptors, most likely the P2XR7 subtype, by activating multiple signaling pathways including the ERK/p38 cascade [19]. Major inflammatory pathways of TNF and NF-KB signaling were studied in the context of apoptosis, cell survival, inflammation, and immunity [20]. However, while some molecular entities in these signal transduction cascades are widely expressed, others are microglia-specific. For example, TNF- $\alpha$ receptor Tnfrsf1b is highly expressed in microglia while the major receptor Tnfrsf1a, is expressed ubiquitously. The mode of TNF- $\alpha$ receptor binding determines activation of the JNK pathway, nuclear translocation of NF-KB, and the recruitment of PI3K/AKT pathway [21-24].

In this study, we applied RNA sequencing analysis (RNA-seq) to quantify gene expression and to examine the molecular changes occurring in a primary neonatal microglia culture following an external stimulus. Specifically, we monitored the dynamics of the transcriptomic profile and cytokine release in response to activation by LPS with and without the addition of benzoyl ATP (bzATP), an agonist of the P2RX7 subtype of ATP receptors. The detailed waves of expression reveal the extent of the condition-dependent response of primary microglia culture. 


\section{Materials and Methods}

Compounds and reagents. Dulbecco's Modified Eagle Medium (DMEM), DMEM/F12, Gentamycin sulfate and L-Glutamine were obtained from Biological Industries (Beit-Haemek, Israel), and 2'-3'-O(4-benzoyl benzoyl) adenosine 5'-triphosphate (bzATP), Bovine serum albumin (BSA) and LPS were purchased from Sigma-Aldrich (Israel).

Preparation of microglial cultures. Primary microglia were isolated from the brains of neonatal male Balb/C mice (Harlan Sprague Dawley, Inc., Israel) as described in [25]. The cells were isolated from the cortex and additional structures and plated in Poly-L-lysine coated flasks for one week. Following an enzymatic dissociation protocol, the non-adherent and loosely adhered cells were re-plated for 1 hour (hr) on bacteriological plates, which allowed sorting out cells exhibiting a slower kinetic of adherence. Microglial cells were propagated by supplementing the culture with $10-20 \%$ of medium conditioned from L-cells that produce mouse-CSF (colony-stimulating factor). Under such conditions, the microglial culture remains responsive for four weeks. Before conducting experiments, the microglial cells were removed from the conditioned medium for $24 \mathrm{hrs}$. In all experiments the heat-inactivated fetal calf serum (FCS) was replaced by purified BSA. The purity of microglia was confirmed by immunostaining and morphological criteria to distinguish them from astrocytes and oligodendrocytes. Distinct morphology and staining by P2Y12, F4/80, CR3 (complement receptor-3), and Galectin-3/MAC-2 validated the purity of the culture to be $>95 \%$ as shown in [26].

Measurement of cytokines. Activation of the cultured microglia was measured by cytokine release as previously described [27] and according to manufacturer's protocols. Cells were grown to $75 \%$ confluence in 6-well plates. Measurements of cytokine secretion were made 8 and $24 \mathrm{hrs}$ after activation in the presence of BSA $(0.4 \mu \mathrm{M})$ using Max deluxe (Biolegend, CA, USA) ELISA kits [28]. Microglia were stimulated by bzATP $(400 \mu \mathrm{M})$, LPS $(1 \mu \mathrm{g} / \mathrm{ml})$ and their combination. The protein content of the cells was measured using BCA Protein Assay (Pierce). Assays were repeated with an internal control for cytokines for $5 \times 10^{5}$ cells per well. Cells were harvested by scraping with a rubber policeman, washed with $\mathrm{PBS}\left(4^{\circ} \mathrm{C}\right)$ and counted. We obtained $40 \mu \mathrm{g}$ lysate from $5 \times 10^{5}$ cells following Iysis and clearance by centrifugation $\left(14,000 \mathrm{~g}, 10 \mathrm{~min}, 4^{\circ} \mathrm{C}\right)$. Each cytokine assay was calibrated by an internal standard curve.

RNA-seq. Microglial cultures were harvested using a cell-scraper. Total RNA was purified from $10^{6}$ cells using QIAzol Lysis Reagent RNeasy plus Universal Mini Kit (QIAGEN, GmbH, Hilden, Germany). To ensure homogenization, a QIAshredder (QIAGEN, GmbH, Hilden, Germany) mini-spin column was used. Samples were transferred to a RNeasy Mini spin column and centrifuged for $15 \mathrm{~s}$ at $\geq 8000 \mathrm{~g}$ at 
room temperature. The mixture was processed according to the manufacturer's standard protocol. Samples with an RNA Integrity Number (RIN) $>8.5$, as measured by Agilent 2100 Bioanalyzer, were considered for further analysis. Total RNA samples ( $1 \mu \mathrm{g}$ RNA) were enriched for mRNAs by pull-down of poly $(A)^{+}$RNA. RNA-seq libraries were prepared using the KAPA stranded RNA-seq kit (Roche) according to the manufacturer's protocol and sequenced using Illumina NextSeq 500 to generate 85 bp single-end reads.

Bioinformatic analysis and statistics. All next-generation sequencing data underwent quality control using FastQC [29] and were processed using Trimmomatic [30], aligned to GRCh38 using STAR. All genomic loci were annotated using GENCODE version 32 [31]. Trimmed Mean of M-values (TMM) normalization of RNA read counts and differential expression analysis were performed using edgeR [32]. The low variability of the TMM values within a triplicate group confirms the quality of the RNAseq data. For differential expression (DE) analysis, additional filtrations with FDR q-value $<0.05$ and a minimal expression level of >10 TMM for the average of naïve and bzATP treated cells were applied. The partition of DE genes to clusters was done according to threshold listed in Table 1.

Table 1. Thresholds used for assigning DE genes for 9 clusters with distinct expression trends.

\begin{tabular}{|c|c|c|}
\hline Trend & 3 hours $(\log F C)$ & 8 hours (logFC2) \\
\hline$\uparrow \uparrow$ & $\log F C>0.5$ & $\log F C 2 \geq \log F C+0.5$ \\
\hline$\uparrow \approx$ & $\log F C>0.5$ & $\log F C 2 \leq \log F C+0.5 \& \log F C 2 \geq \log F C-0.5$ \\
\hline$\uparrow \downarrow$ & $\log F C>0.5$ & $\log F C 2<\log F C-0.5$ \\
\hline$\approx \uparrow$ & $-0.5 \leq \log F C \leq 0.5$ & $\log F C 2>\log F C+0.5$ \\
\hline$\approx \approx$ & $-0.5 \leq \log F C \leq 0.5$ & $\log F C 2 \leq \log F C+0.5 \& \log F C 2 \geq \log F C-0.5$ \\
\hline$\approx \downarrow$ & $-0.5 \leq \log F C \leq 0.5$ & $\log F C 2<\log F C-0.5$ \\
\hline$\downarrow \uparrow$ & $\log F C<-0.5$ & $\operatorname{logFC2}>\log F C+0.5$ \\
\hline$\downarrow \approx$ & $\log F C<-0.5$ & $\log F C 2 \leq \log F C+0.5 \& \log F C 2 \geq \log F C-0.5$ \\
\hline$\downarrow \downarrow$ & $\log \mathrm{FC}<-0.5$ & $\log F C 2<\log F C-0.5$ \\
\hline
\end{tabular}

$\uparrow$ up, $\downarrow$ down, $\approx$ unchanged.

Pathway and gene-set enrichment analyses were performed using the clusterProfiler: enrichKEGG program and the pathview for visualization. GO annotation enrichment was based on GOrilla statistical tool [33]. Protein protein interaction map was based on high scoring STRING interactome (score >0.9) [34]. ID conversion from Ensemble to Entrez was carried out by the annotation package (biomaRt, org.Mm.eg.db). Experiments contained a minimum of three biological replicates. The cytokine quantification data were analyzed by one-way analysis of variance (ANOVA), using IBM SPSS Statistics Version 19 followed by Duncan's post hoc test. Results from microglial cell experiments are presented as mean \pm SD (standard deviation). All other statistical tests were performed using R-base functions. When appropriate, $p$-values $<0.05$ were calculated and considered statistically significant. Principal component analysis (PCA) was performed using the R-base function "prcomp". For selection 
of top 1000 genes (10\% of all reliable expressed genes) we have used plotMDS (multidimensional scaling plot of distances between gene expression profile). Figures were generated using the ggplot2 R package.

Gel based RT-PCR. Reverse transcription polymerase chain reaction (RT-PCR) assays followed by a gelbased separation was performed for selected genes. cDNA was prepared by the High Capacity cDNA Reverse Transcription Kit (ThermoFisher) according to the manufacturer's instructions, using RT random primers, and MultiScribeTM Reverse Transcriptase. For PCR reaction we have used the PCRBIO-HS Taq mix (PCRBiosystem; London, UK) according to the recommended protocol that consists of a denaturation step at $95^{\circ} \mathrm{C}(2 \mathrm{~min})$ followed by 35 cycles $\left(10 \mathrm{sec}\right.$ at $95^{\circ} \mathrm{C}, 15 \mathrm{sec}$ annealing at $60^{\circ} \mathrm{C}$, and $10 \mathrm{sec}$ extension for at $72{ }^{\circ} \mathrm{C}$ ). The tested genes are: 116 (NM_031168), Fw: 5'CACTTCACAAGTCGGAGGCT; Rev: 5'GGAGAGCATTGGAAATTGGGG (380 nt); Tnf (NM_013693), Fw: 5'ACAGAAAGTCATGATCCGCGA; Rev: 5'GTTTGCTACGACGTGGGCT (288 nt); Il1a (NM_010554), Fw: 5'AGGGAGTCAACTCATTGGCG; Rev: 5'ACTTCTGCCTGACGAGCTTC (449 nt); Il1b (NM_008361), Fw: 5'TGCCACCTTTTGACAGTGATG; Rev: 5'GGAGCCTGTAGTGCAGTTG (351 nt); Tnfrsf1b (NM_011610), Fw: 5'CACTTGGGGCCGACTTGTTA; Rev: 5'CCGTCTCCTTCCCACAACAC (445 nt). $\beta$-actin, Fw:

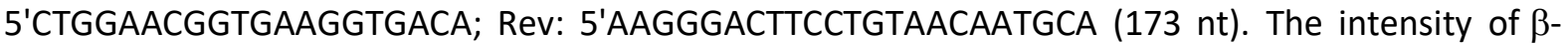
actin was used as a control.

\section{Results}

The functional response of primary microglia. The function of the microglia cells was monitored by quantifying the secretion of TNF- $\alpha$ and IL-6 following activation with bzATP (Fig. 1A). Secreted cytokines were below the level of detection prior to the addition of bzATP/LPS ( $<5 \mathrm{pg} / \mu \mathrm{g}$ of cell lysate) but increased markedly 8 and 24 hrs after simulation. While the amount of IL-6 secreted were larger when collected over a period of $24 \mathrm{hrs}$ than at $8 \mathrm{hrs}$ ( $p$-value $<0.0001$ ), those of TNF- $\alpha$ was reduced ( $p$-value <0.001) (Fig. 1A). Moreover, 8 hrs following stimulation for absolute amounts of IL-6 and TNF $\alpha$ secreted were 5.92 and $423.4 \mathrm{pg} / \mu \mathrm{g}$ of microglial cell lysate, respectively. These results implying that both the kinetics and amounts of secreted cytokines were drastically different.

We then measured the transcript levels of Tnf and 116 in naïve cells and upon bzATP/LPS stimulation. We noted that Tnf (gene product TNF $\alpha$ ) level can be detected in the naïve cells. This level was strongly increased at $3 \mathrm{hrs}$ and declined at $8 \mathrm{hrs}$ of bzATP/LPS. In quantitative terms, the Tnf transcript accounts for $0.02 \%, 1.2 \%$, and $0.36 \%$ of all transcripts for naïve cells, 3 and 8 hrs after bzATP/LPS, respectively. In contrast, the mRNA of 116 is below detection in naïve cells, and its induction 
is relatively slow (Fig. 1B). We conclude that gene expression of TNF release is stimulus-dependent. IL-6 secretion displayed a continuous elevation of the secreted cytokine at 24 hrs post-stimulus (Fig. 1A) which agrees with the upregulation of the 116 transcript. The slow kinetics of IL-6 release compared to that of TNF- $\alpha$ in microglial cells confirms the report of [35], implying a distinct activation route for each of the cytokines.
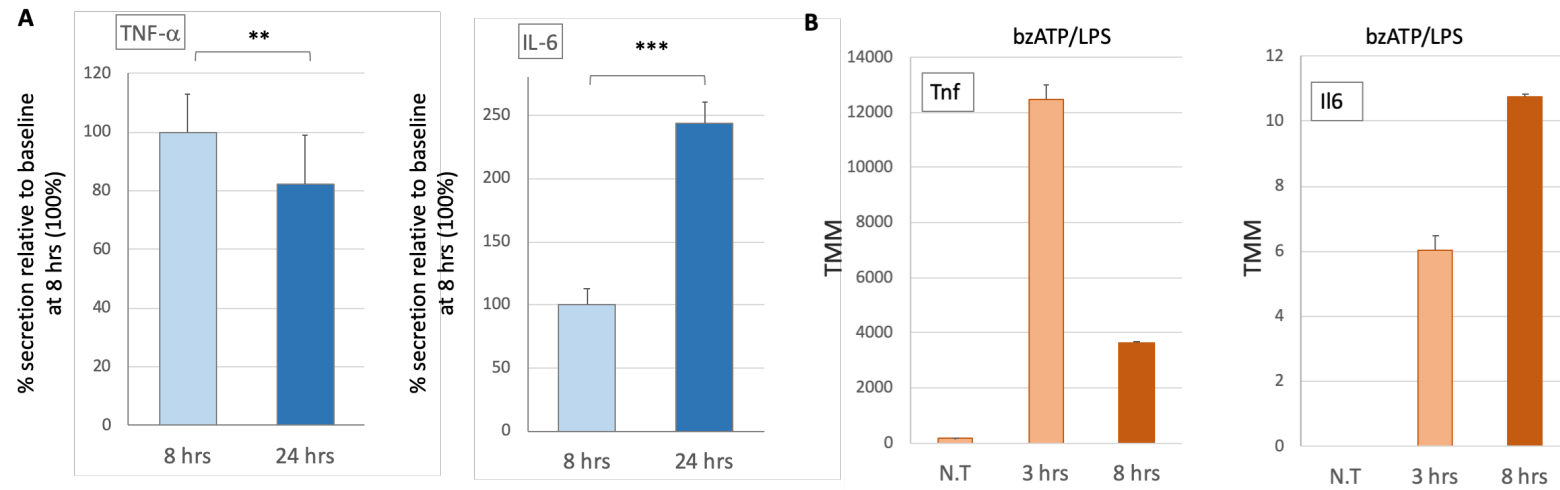

Figure 1. Quantitation of TNF- $\alpha$ and IL-6 released from primary neonatal murine microglial culture. (A) Values are normalized with respect to protein concentrations ( $\mu$ g lysate) from the adhered cultured cells. The levels of TNF- $\alpha$ and IL- 6 were below detection in naïve unstimulated cells. Samples for the analysis were derived from the conditioned media supplemented with BSA, harvested, and measured at 8 and $24 \mathrm{hrs}$ after stimulation. A mean and standard deviation (s.d.) of 4 experiments are shown (each group, $\mathrm{N}=22-24)$. Statistical significance marked by asterisks implies the results of Mann Whitney test p-value of $0.001\left(^{* *}\right)$ and $<0.0001\left(^{* * *}\right)$. (B) Kinetics of Tnf and 116 transcripts upon bzATP/LPS stimulation based on RNA-seq data. Results are the average of biological triplicates. The 116 in not expressed in naïve cells (N.T., not treated).

A short-lived transient wave of gene expression by bzATP. To establish the characteristics of microglia and to quantify the molecular events that occur on changing cells from their resting state to maximal activation, we first tested the cells' transcriptome at $3 \mathrm{hrs}$ and $8 \mathrm{hrs}$ following exposure to bzATP. Using RNA-seq analysis, we report 10,835 genes were expressed that complied with the statistical quality threshold (see Materials and Methods).

Fig. 2 shows the partition of these genes into 9 clusters along with their fold change (FC). The expression levels of almost all these genes (96\%) are unchanged and thus are labeled 'Same' (Fig. 2A). The term 'Same' marks changes in expression that are bounded by $50 \%$ (i.e., at a 0.67 to 1.5 -fold difference relative to genes expressed in naïve cells). Fig. 2B shows the partition of genes in the presence of bzATP for $3 \mathrm{hrs}$. Notably, at $8 \mathrm{hrs}$, most genes were still marked as 'Same' (Fig. 2B, gray color). The relative expression of the genes belonging to the 9 groups is shown in Fig. S1.

Next, we tested the validity of the above observations for a filtered list of genes with strict thresholds. To satisfy a false discovery rate (FDR) of q-value $<0.05$ and an expression level $>10$ TMM (see Materials and Methods), about $67 \%$ of the original gene list was eliminated. We observed that 
the vast majority of the filtered DE genes (93\% of 3578 genes) are labeled 'Same', with only 16 genes (0.5\%) significantly changing their expression in a monotonic trend (i.e., labeled Up-Up and DownDown; Table S1).
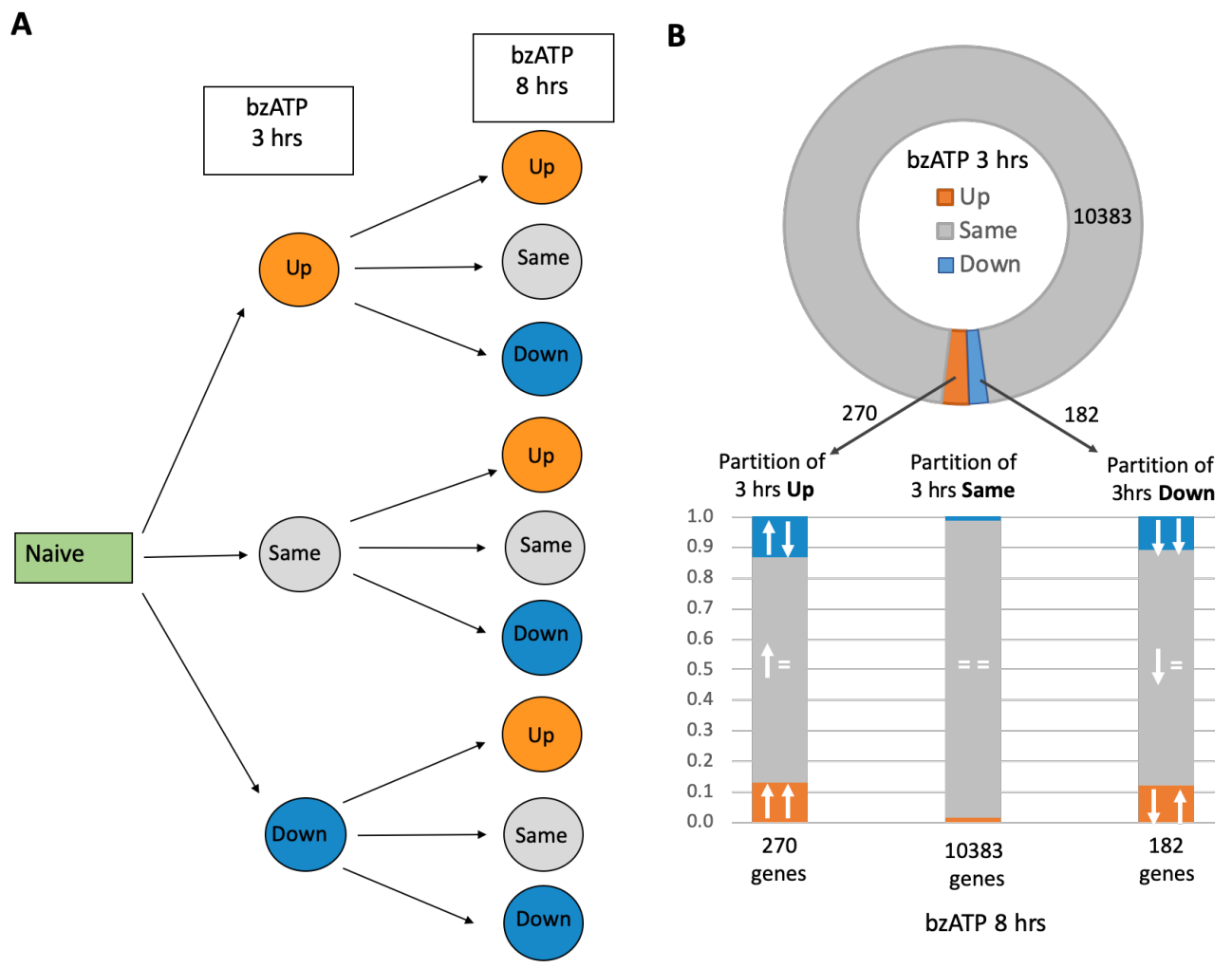

Figure 2. Trends in gene expression of microglial genes in the naïve (not treated) and following activation protocol with BzATP for $3 \mathrm{hrs}$ and $8 \mathrm{hrs}$. (A) Differentially expressed genes are partitioned to 9 groups according to the joined expression trend (Up, Down or Same, and their combinations). (B) The ring-shaped graph shows the partition of the 10,835 expressed genes by their expression trend at $3 \mathrm{hrs}$ of bzATP treatment relative to naïve cells. The number of genes labeled as Up, Same, Down is indicated. The histogram shows the partition of each of the three clusters according to the partition of expression trend measured at $8 \mathrm{hrs}$ following bzATP exposure. Symbols for up ( $\uparrow)$, down $(\downarrow)$ and same $(=)$ are shown. The analysis is based on data in Table $\mathbf{S 1}$.

Fig. 3 shows the downregulated (7 genes, FDR q-value ranges from 3.1e-18 to 2.9e-10; Fig. 3A) and upregulated DE genes (9 genes, FDR q-value ranges from 2.7e-18 to 5.6e-04; Fig. 3B). Among the genes in Fig. 3A that are continuously suppressed are the enzyme hyaluronidase (Hyal1) and semaphorin receptor (Sema4d, also called Cd100) that contribute to cell migration and differentiation. In contrast, the inflammatory signal dominates the genes in Fig. 3B. For example, Trem1 is a scaffold membrane protein that acts in chemotaxis and regulates the killing of Gram-negative bacteria. Additional genes belong to chemokine ( $\mathrm{Cxcl} 2$, chemokine $\mathrm{C}-\mathrm{X}-\mathrm{C}$ motif ligand 2 ) and their immediate pathways (II18rap, II1a, IL1b). We conclude that exposing murine microglia to bzATP led to a transient 
wave of gene expression which faded out at a longer timeframe ( $8 \mathrm{hrs})$. Moreover, this exposure activates a distinctive set of genes that act as sensors for the immune-related response of microglia.

A

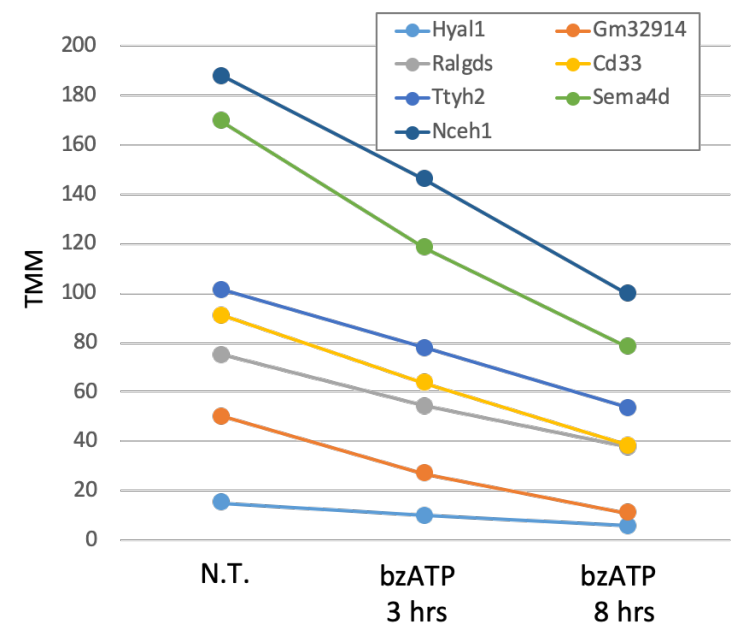

B

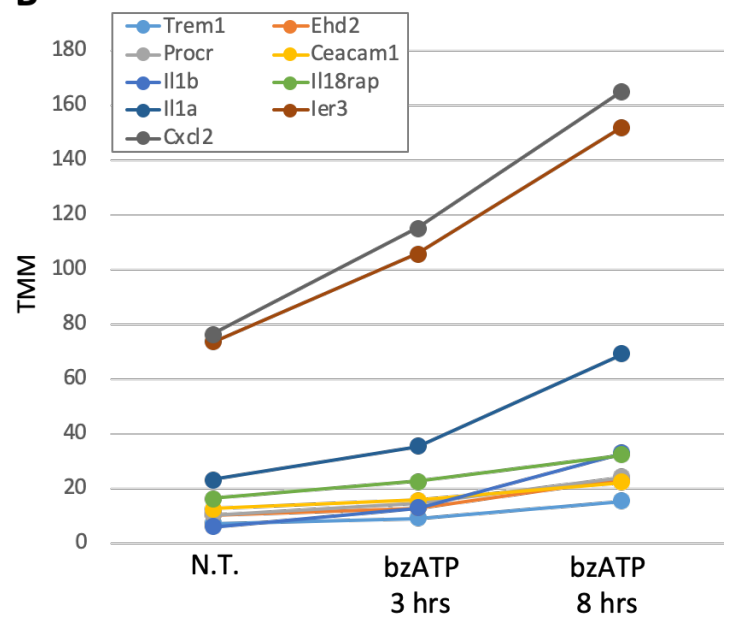

Figure 3. Dynamics of differential expressed (DE) genes following activation with bzATP. DE genes in naïve cells (N.T., not treated) and following bzATP treatment for $3 \mathrm{hrs}$ and $8 \mathrm{hrs}$. The reported genes meet strict thresholds of FDR (q-value $<0.05$ ) and minimal expression level (see Materials and Methods). (A) Set of genes with a continuously reduced expression (labeled Down-Down). (B) Set of genes with upregulated expression (labeled Up-Up). The analysis is based on data in Table $\mathbf{S 1 .}$

Genes that respond to bzATP with slow kinetics regulate microglial communication. In addition to DE genes with a monotonic trend in expression (Fig. 3), we investigated a set of genes with slow kinetics (i.e., did not change significantly in 3 hrs but only after 8 hrs). Supplemenrary Table S2 lists 26 of the most significant such DE genes (at a strict FDR $<1.0 \mathrm{e}-10$ for the kinetics of 3 hrs vs. 8 hrs; $T M M>10)$. Genes that act in trafficking (i.e., Rab7b, Snx7) and cell-cell interaction (e.g., Cadm1, Vwf) are those marked as downregulated at $8 \mathrm{hrs}$. Specifically, Cadm1 mediates homophilic cell-cell adhesion along with neuronal migration. The larger fraction of DE genes with slow kinetics are associated with regulation of immune maintenance via TNF signaling. Tnfrsf1b (tumor necrosis factor receptor superfamily, 1b) and Ltb (Lymphotoxin-beta) promote cytokine cell surface signaling, whereas Itgam directly activates TNF-primed neutrophils, regulates neutrophil migration, and is involved in the production of superoxide ions in microglia (Supplementary Table S1). We conclude that the cell system reaches a new homeostatic state by suppression of trafficking and mostly increasing the sensitivity of the immune responsiveness (upregulated). Nevertheless, the changes in expression with later kinetics are modest $<2$ fold (Table S1).

Expression of IL1 as an indicator of microglial activation. The function of cultured neonatal microglia was tested by measuring the release of the pro-inflammatory cytokines (Fig. 1A). As interleukins (IL) 
and their receptors are indicators of the brain inflammatory state, we assessed the relative expression of IL-gene transcripts (33 expressed genes, collectively called IL-gene set; Supplementary Table S3). The results from the RNA-seq showed that IL-1 $\beta$ mRNA increases 4.2 fold within 3 hrs and 10.8 fold 8 hrs after exposure to bzATP (Fig. 4A). Despite this high increase in expression, IL-1 $\beta$ accounts for only $2 \%$ of the total TMM of the IL gene set (considered the TMM of the IL-gene set as $100 \%$ ). By contrast, IL-1 $\beta$ mRNA levels increase 2100 fold within 3 hrs in bzATP/LPS (Fig. 4B), accounting for $35 \%$ of the mRNAs of the IL-gene set. Interestingly, the kinetics of II1a is very similar to that of II1b. Specifically, in the condition of bzATP/LPS, the IL-1 $\alpha$ mRNA level was increased $\sim 500$ fold within 3 hrs, accounting for $48 \%$ of the total IL-gene set in the activated cells. Semi-quantitative RT-PCR assays substantiated the strong induction of transcription of major interleukins. It also agreed with the slow kinetics observed for II6 (Fig. 4C). Unlike interleukins, the receptor II1rl1 increased 9-fold, 3 hrs after activation by BzATP/LPS with no further increase at a later timepoint ( $8 \mathrm{hrs}$; Fig. 4D). The short-lived wave of bzATP activation is seen by focusing on the expression of IL receptors. The IIIrn (Interleukin 1 receptor antagonist), which inhibits the activities of IL- $1 \alpha$ and IL1 $\beta$ is induced transiently by bzATP (1.8 fold, 3 hrs) but returns to its baseline levels at $8 \mathrm{hrs}$. The addition of LPS leads to a substantial and permanent increase in its expression (13.1 and 10.7 fold in 3 and 8 hrs, respectively, Fig. 4D).

The focus on the Interleukins and their receptors sheds light on LPS-induced suppression of expression. In the naïve cells, II7ra (interleukin 17 receptor A) accounts for $30 \%$ of interleukin transcripts. The exposure of cells to bzATP increased the level by $25 \%$, and LPS suppressed the expression to the same extent (25\% of its basal level; Fig. 4D). The level of expression of the II17 receptor in these cells argues against its contribution to the cell activation program. For a detailed gene expression of the IL gene set, see Supplementary Table S3.

We performed PCA analysis (Fig. $4 \mathrm{E}$ ) to assess the coherence of the transcriptional programs identified by the RNA-seq. Clustering experimental samples ( 15 samples, triplicates) into 5 tight groups confirmed that the variance within each group is negligible relative to the variance among the tested conditions (i.e., NT, bzATP, and bzATP/LPS in two-time points). While the actual position in the 2dimensional representation is somewhat arbitrary, PCA results also indicate the relatively restricted impact of bzATP along with a strong and persistent effect induced by bzATP/LPS. Moreover, the delayed kinetics supports the consolidation of the entire transcriptome. The variance explained by the two principal components (PC1, PC2) is $68.6 \%$. Performing a dimension reduction for only 1000 top genes using the multidimensional scaling method (MDS) resulted in a plot that explains $92 \%$ of the variance between PC1 (79\%) and PC2 (13\%; Fig. S2). These results confirm the robustness of the transcriptomic signal from all experimental conditions. 
A

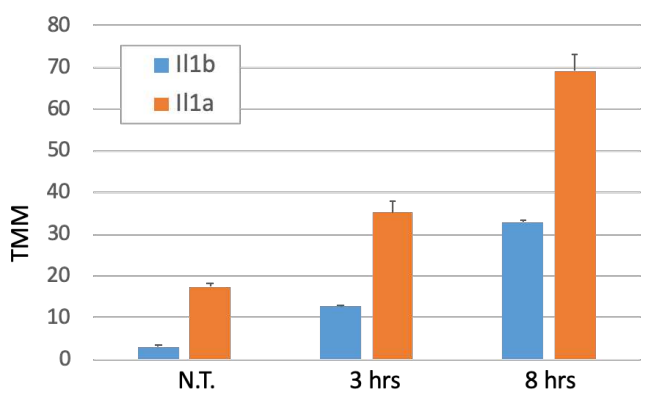

C

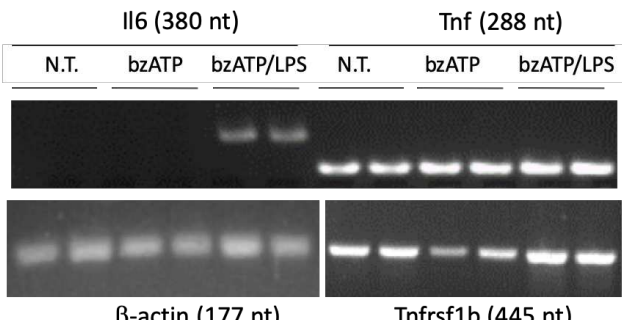

$\beta$-actin (177 nt)

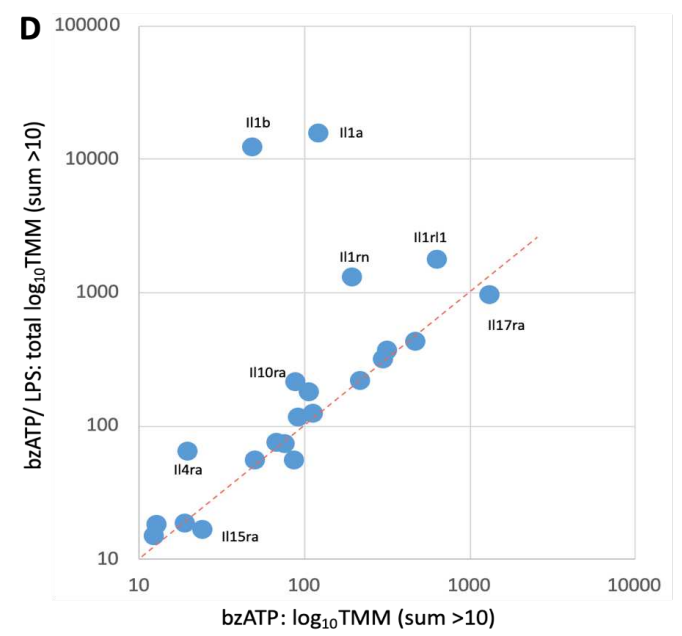

B
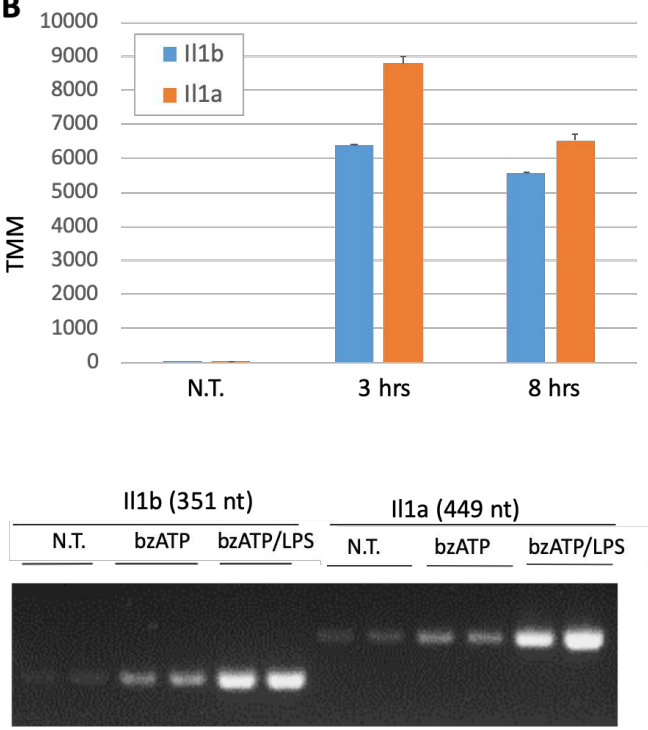

E

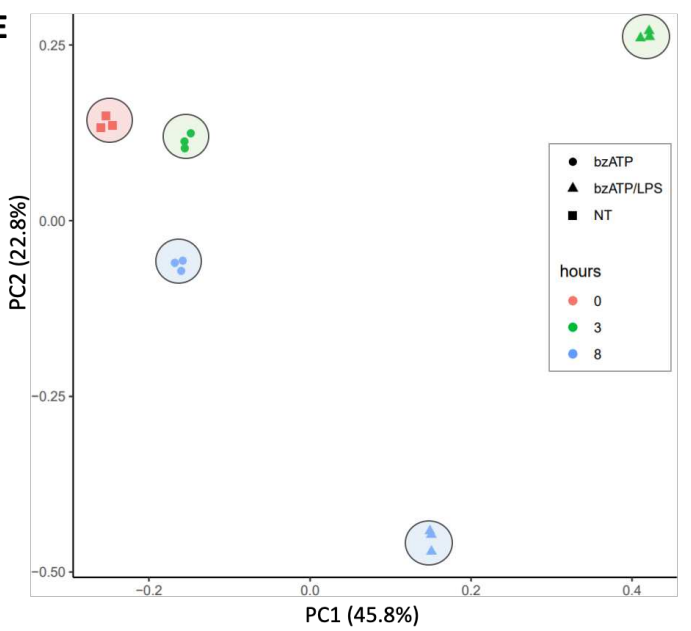

Figure 4. Dynamics of differential gene expression of interleukins and their direct regulators (IL-gene set) in naïve (N.T., not treated) and following treatments for $3 \mathrm{hrs}$ and $8 \mathrm{hrs}$. (A) bzATP. (B) The levels of expression of Il1a and II $1 \mathrm{~b}$ following activation with bzATP/LPS. Note the difference in the $y$-axis. The SDs (standard deviations) are based on the triplicates from RNA-seq. (C) Results from RT-PCR on N.T. cells and cells after exposure to bzATP and bzATP/LPS for $8 \mathrm{hrs}$ (in biological duplicates). The levels of expression of Tnf and Tnfrsf1b are already substantial prior to cell activation. $\beta$-actin serves as an internal control. (D) Analysis of 33 IL-gene set under activation condition by bzATP ( $x$-axis) and bzATP/LPS following $8 \mathrm{hrs}$ ( $y$-axis). Only genes with mean expression levels $>10$ TMM are shown. (E) PCA analysis of the 15 samples of RNA-seq. The variance explained by the two principle components (PC1, PC2) is 68.6\%.

Global alteration of cell transcriptome by bzATP/LPS is synchronized and long-lasting. Fig. 5A shows the partition of the DE genes (total of 10,769 genes) into 9 clusters in the presence of bzATP/LPS. A marked difference in the treatment of bzATP and bzATP/LPS is seen by comparing Fig. 2A and Fig. 5A. bzATP/LPS drastically changed the expression of a large fraction of the genes (44.3\%) within 3 hrs 
(1924, 2851 for Up and Down labeled genes, respectively; Fig. 5A). A detailed list of all 10,769 genes before (time $=0$ ) and $3 \mathrm{hrs}$ and $8 \mathrm{hrs}$ after activation is shown in Fig. $\mathbf{S 3}$.
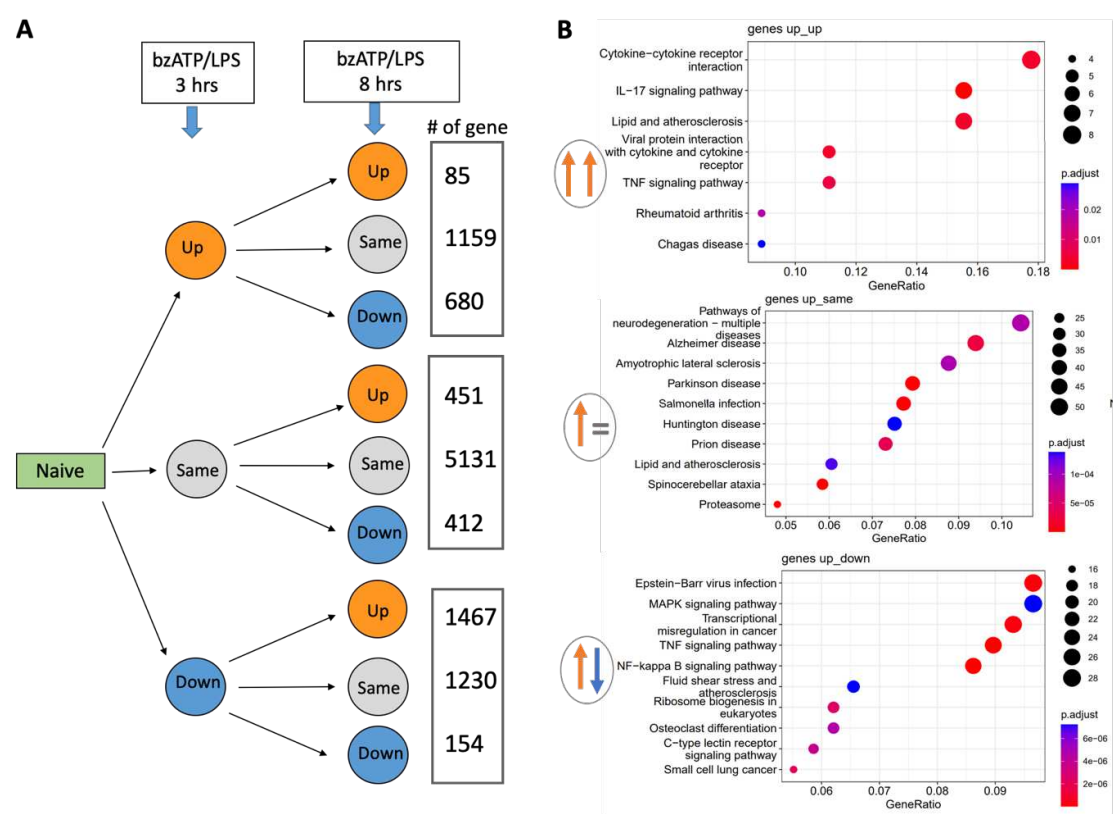

C

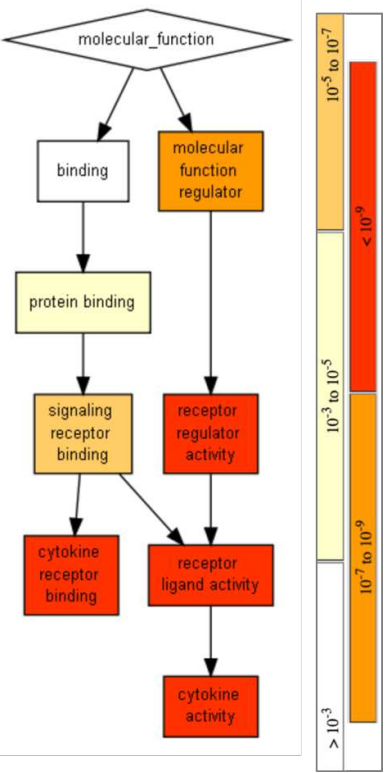

Figure 5. Partition of genes by their expression trends and their functional enrichment. (A) Gene expression levels of naïve microglial cells and following activation with bzATP/LPS for $3 \mathrm{hrs}$ and $8 \mathrm{hrs}$. A scheme of all 9 expression trends for 10,769 genes, together with the number of genes associated with each cluster is shown. For details see Supplementary Table S4. (B) Enrichment analysis for DE genes for genes induced after 3 hrs (i.e., labeled Up). Annotations are according to KEGG pathway database. Statistical enrichment of $p$-adjust is depicted by the colors (blue to red) for FDR $<0.05$. The size of the dots captures the number of proteins. Note that the number of genes associated with each enriched pathway, and the gene ratio (i.e., the fraction of genes in the cluster that are included in a specific pathway, $x$-axis) differ among gene clusters. (C) Enrichment analysis for $\mathrm{GO}$ annotations of upregulated DE genes. Analysis performed on strongly upregulated genes (total 585 genes, $>1.5$ fold increase in expression relative to non-treated cells, average expression level >10 TMM). The enrichment results for GO molecular function satisfied p-value $<1.0 \mathrm{e}-10$ for the analysis. Color codes of white to red (right) indicates the adjusted-p-values after multiple testing corrections.

Fig. 5B shows the cellular pathways (by KEGG) enrichment for the genes upregulated by bzATP/LPS at $3 \mathrm{hrs}$. The partition of these genes (total 1924) by their expression trend (Up-Up, UpSame, and Up-Down) is shown. In the cluster labeled Up-Up (85 genes), the listed pathways are associated with cytokines (e.g., TNF signaling), viral infection, and inflammation-based diseases. By contrast, the cluster marked as Up-Same (1159 genes) showed enrichment in aging and degenerative CNS diseases. The Up-Down cluster (680 genes) is enriched in signaling pathways such as MAP kinase, $\mathrm{TNF}$, and NF-KB. We conclude that many of the enriched pathways overlap with an immunological response, thus confirming the nature of a robust transcriptional wave. The enrichment analyses for the nine expression trends are available in Supplementary Fig. S3.

We further analyzed the DE genes that were upregulated by bzATP/LPS stimulation according to the gene ontology (GO) annotations (Fig. 5C). We focused on the statistically significant DE genes that 
were strongly induced. Inspection of the molecular function revealed a significant enrichment of cytokine activity and cytokine receptor binding. Repeating the GO enrichment test by using a more relaxed threshold ( $p$-value $<1.0 e-07$ ), confirmed the previously identified annotations (i.e., cytokine regulation), and exposed additional terms for kinase phosphorylation (Supplementary Fig. S5). We conclude that an unbiased view of global gene expression supports a robust and long-lasting induction of cytokines and chemokines.

Changes in gene expression by orders of magnitude drive the microglia's activation state. To assess the kinetics and extent of the transcriptional waves we focused on the DE genes according to their fold ratio increase in the presence of bzATP/LPS to that in naïve cells ( 8 hrs). Fig. 6 shows the sorted list of 25 such genes. Already after $3 \mathrm{hrs}$, the degree of induction for this small set of genes is substantial and remains high for most genes at 8 hrs (Figs. 6A, 6B). The upregulation ranges from 5.5 to 2800 folds (Fig. 6 C) and the majority of the proteins encoded by these genes are functionally and physically connected (Fig. 6D). Among the highly connected proteins are Tnf and $\| 1 \mathrm{~b}$, and components of the chemokine signaling ( $\mathrm{Cxcl} 10, \mathrm{Ccl} 2, \mathrm{Cxcl} 2, \mathrm{Ccl}$, $\mathrm{Csf} 2)$.

We concluded that coordinated and extremely strong transcriptional induction governs the activation state of the microglial culture. This is further confirmed by inspecting the most significant downregulated genes (>10 TMM). Altogether, the expression of 720 genes was downregulated by a factor of 2-20. These genes failed to indicate any significant enrichment using GO annotation analysis and functional connectivity or KEGG pathways (Supplementary Fig. S4). Of special interest is P2RX7, the ATP-gated cation channel that participates in neuro-inflammation and pathophysiological processes. The P2rx7 gene transcript showed a suppressed expression by fast kinetics (by 8.3 fold, 3 hrs after the addition of bzATP/LTP), with partial recovery at $8 \mathrm{hrs}$ and supports a previous. observation by [28]. We conclude that while hundreds of genes are up- and downregulated as a result of the stimuli, only a selected set that was upregulated by orders of magnitude drive the robust inflammatory response. 
A
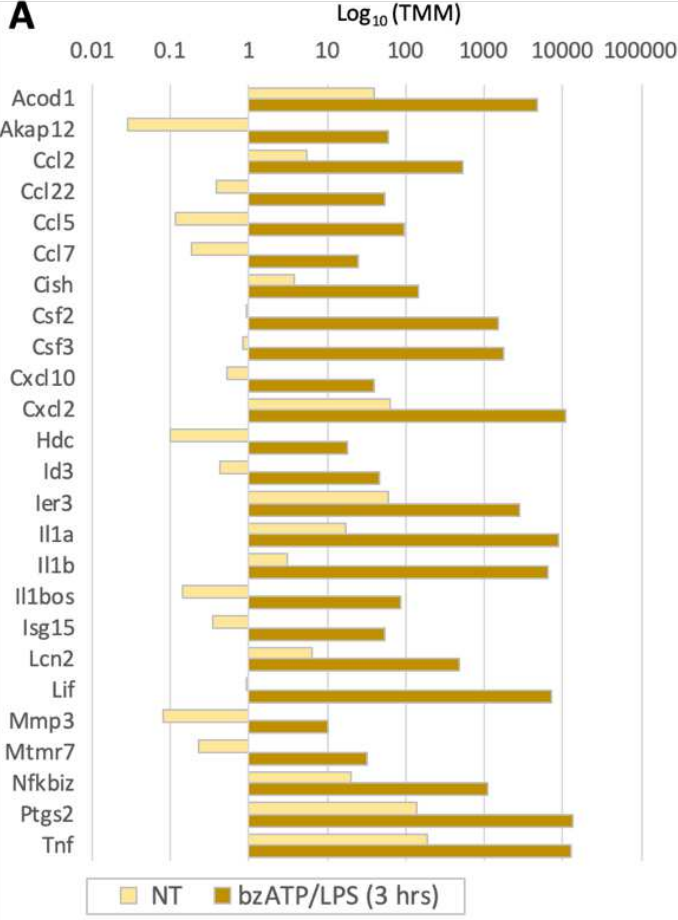

C

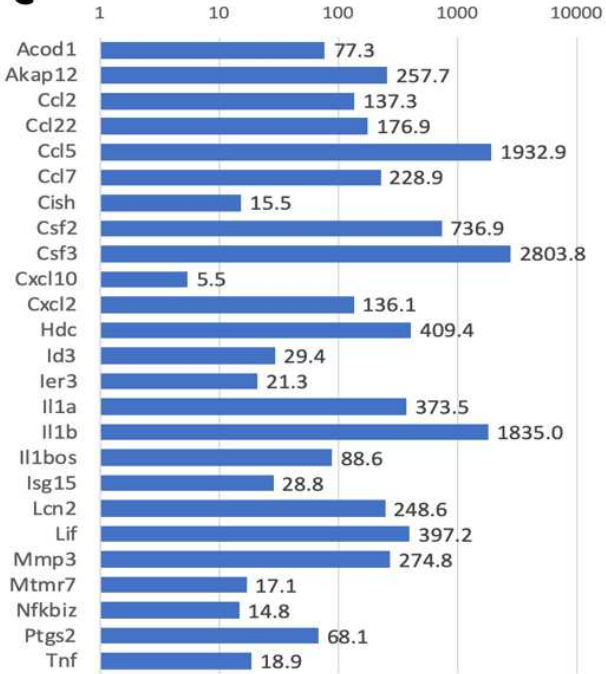

B
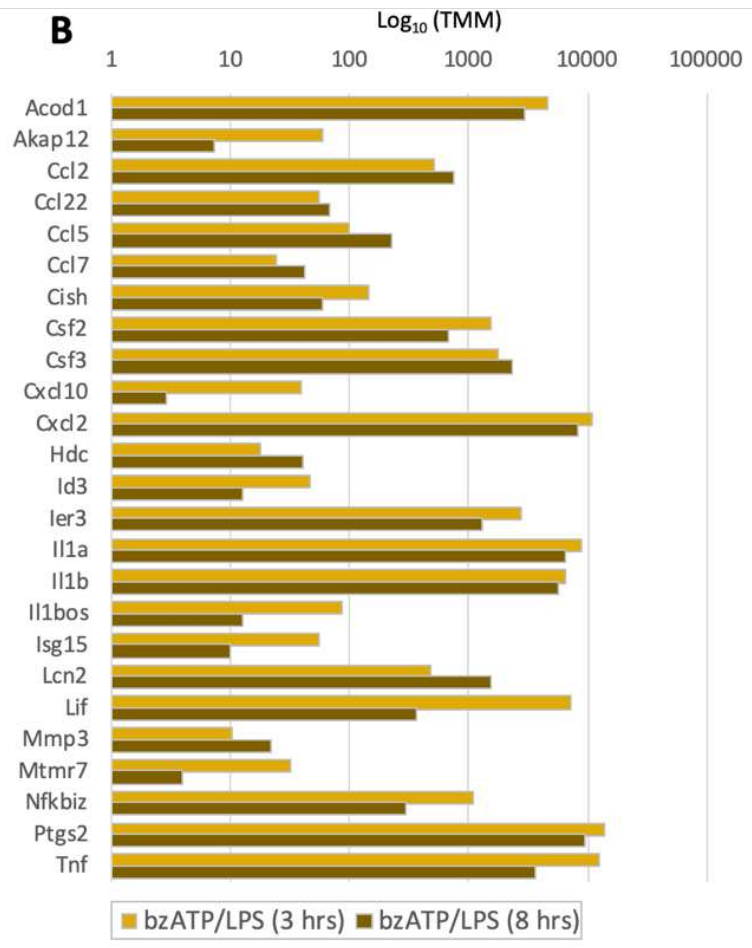

D

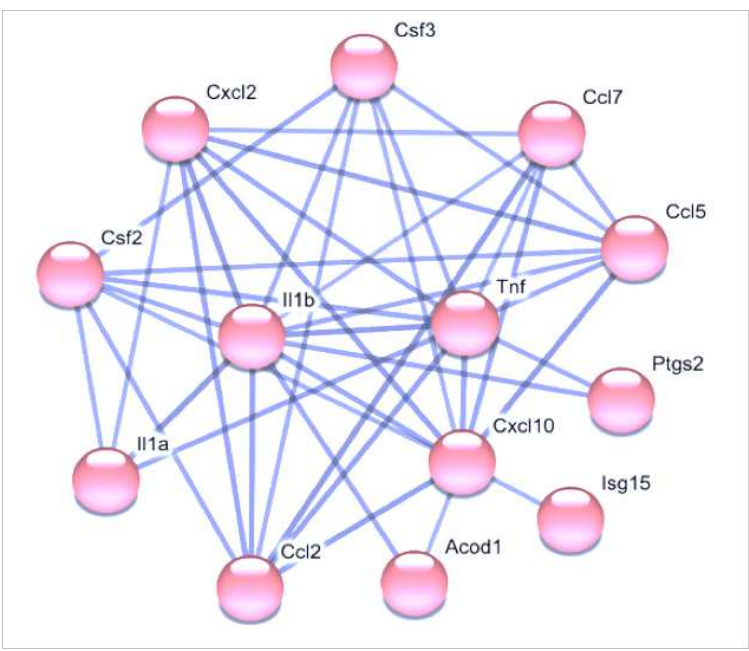

Figure 6. Top 25 induced genes sorted by their extent of induction by bzATP/LPS after 8 hrs relative to naïve cells. (A) $\log _{10}$ TMM of the expression in naïve, non-treated cells (NT) and $3 \mathrm{hrs}$ of bzATP/LPS. (B) $\log _{10}$ TMM of the expression in $3 \mathrm{hrs}$ and $8 \mathrm{hrs}$ following bzATP/LPS stimulation. The genes in A-C are listed alphabetically. (C) Increase at $8 \mathrm{hrs}$ relative to that in naïve cells, e.g., Csf3 (colony stimulating factor 3 ) increases 2803.8 fold. For details see Table S5. (D) A connectivity map according to STRING protein interaction network. Only the genes $(13 / 25)$ with high confidence connection score $(>0.9)$ are shown.

\section{Different signaling pathways associated with microglia activation by bzATP and bzATP/LPS. To see}

whether bzATP activates the same pathways as those in cells exposed to bzATP/LPS, albeit to a lower extent, we used STRING [34] as a protein-protein interaction (PPI) map and projected the most significant DE genes (100 genes; sorted by p-value) that show the largest connected component. Fig. 7 shows the marked difference in the resulting network for each of the experimental conditions. 
A

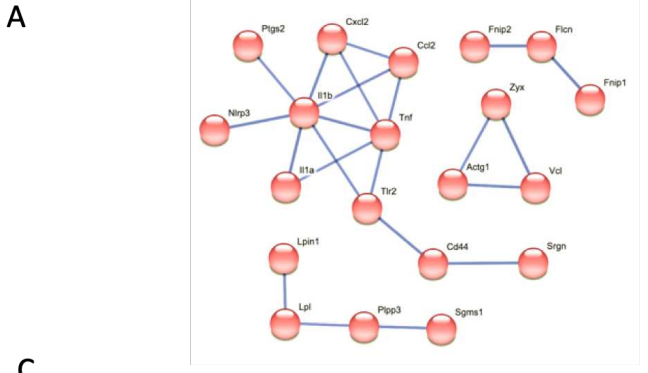

C

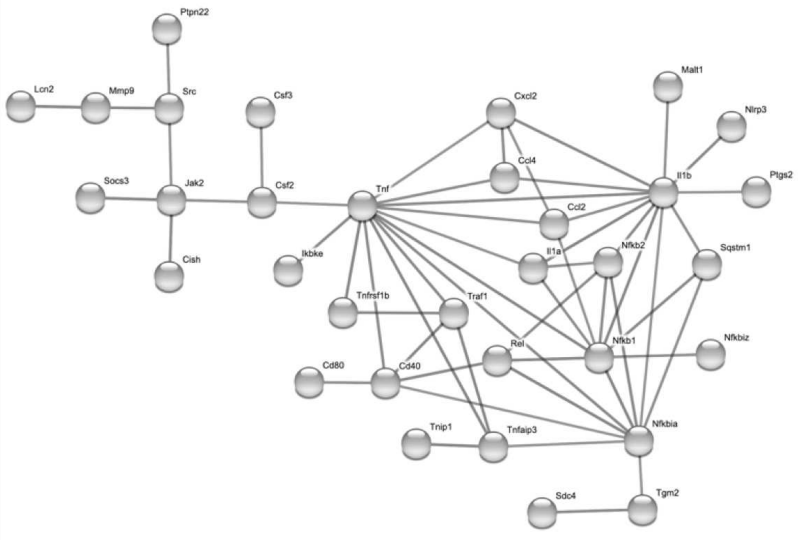

B

\begin{tabular}{lll} 
pathway & description & false discovery rate \\
\hline WP3632 & Lung fibrosis
\end{tabular}

D

\begin{tabular}{ll|l}
\multicolumn{1}{c|}{$\begin{array}{l}\text { pathway } \\
\text { WP246 }\end{array}$} & $\begin{array}{c}\text { description } \\
\text { false discovery rate }\end{array}$ \\
\hline WP3632 & TNF-alpha NF-kB signaling pathw & $5.48 \mathrm{e}-09$ \\
\hline WP222 & Lung fibrosis & $2.03 \mathrm{e}-08$ \\
\hline WP37 & Cytokines and inflammatory resp' & $3.77 \mathrm{e}-06$ \\
\hline WP2432 & IL-1 signaling pathway & $1.01 \mathrm{e}-05$ \\
\hline WP373 & Spinal cord injury & $1.01 \mathrm{e}-05$ \\
\hline WP1496 & IL-3 signaling pathway & $1.01 \mathrm{e}-05$ \\
\hline WP1254 & Oxidative damage response & $1.49 \mathrm{e}-05$ \\
\hline WP493 & Apoptosis & $2.56 \mathrm{e}-05$ \\
\hline WP447 & Mapk signaling pathway & 0.00015 \\
\hline WP1243 & Adipogenesis genes & 0.00050 \\
\hline & EBV LMP1 signaling & 0.00067
\end{tabular}

Figure 7. STRING interaction network for the 100 most significant genes (sorted by DE analysis) for the RNA-seq results for. STRING scoring of high confidence connections $(>0.9)$ and at least 3 connected genes are shown. (A) bzATP treatment (8 hrs). (B) Statistically significant enriched pathways from WikiPathways [36] that satisfies a statistical threshold of False discovery rate (FDR) $<1.0 \mathrm{e}-3$. (C) bzATP/LPS ( $8 \mathrm{hrs}$ ) and (D) the list of most significant pathways that satisfies a statistical threshold of False discovery rate (FDR) $<1.0 \mathrm{e}-3$.

Note a difference between the network for the bzATP ( $8 \mathrm{hrs}$ ) and bzATP/LPS in the size of the network (Fig. 7A, 7C). It includes $10 \%$ and 33\% of the input genes for bzATP and bzATP/LPS conditions, respectively. The enriched pathways (based on WikiPathways [36] in cells treated with bzATP is lung fibrosis (FDR = 9.6e-04, Fig. 7B), while bzATP/LPS condition was strongly enriched with many inflammatory signals (Fig. 7D) including TNF and NF-kappa B signaling (FDR = 5.48e-09), IL-1 and IL-3 signaling $($ FDR $=1.01 \mathrm{e}-05)$, oxidative damage response $(1.49 \mathrm{e}-05)$ and more. We concluded that bzATP/LPS (8 hrs) changes gene expression following by strongly activating numerous inflammatory pathways, most notably is the TNF- $\alpha$ and NF- $\kappa B$ signaling.

Alternative signaling patterns dominate the microglia activation states. We compared our results with RNA-seq with those in microglia isolated from C57BL/6J mice before and after activation with LPS+IFN $\gamma$ [37]. Several of the highest upregulated genes (e.g., Tnf, Csf3, Fig. 8) showed a similar trend in the two experiments. To pinpoint the difference in the signaling cascades in the two settings, we reanalyzed the DE genes while focusing on the PI3K-Akt branch of the TNF signaling map from KEGG [38]. Fig. 8 and Fig. S6 show the expression trend following 8 hrs in bzATP/LPS compared to the activation reported by LPS+IFN $\gamma$ [37]. The TNF signaling pathway shows a cascade from TNF- $\alpha$ binding to the cell surface receptor through the execution of cell survival, apoptosis until induction of 
inflammatory cytokines. Fig. 8 highlights the differences in the expression trend between the two settings. We concluded that changes in gene expression induced by bzATP/LPS are converged to signaling that shows an opposite trend for p38 protein (Mapk14 gene) and Irf1 (Interferon regulatory factor 1). Of the transcription factors, Irf1 is likely a regulator of various pro- and anti-inflammatory states [39] that regulates the release of IFN $\gamma$. Irf1 rapidly enhanced IFN- $\beta$ and IFN- $\gamma$ after stimulation with nucleic acids that mimics vial infection [40, 41]. In accord with this activation specificity, the expression of both Irf1 and INF $\gamma$ was upregulated only in cell culture that was activated by LPS+IFN $\gamma$ but not by bzATP/LPS.

A

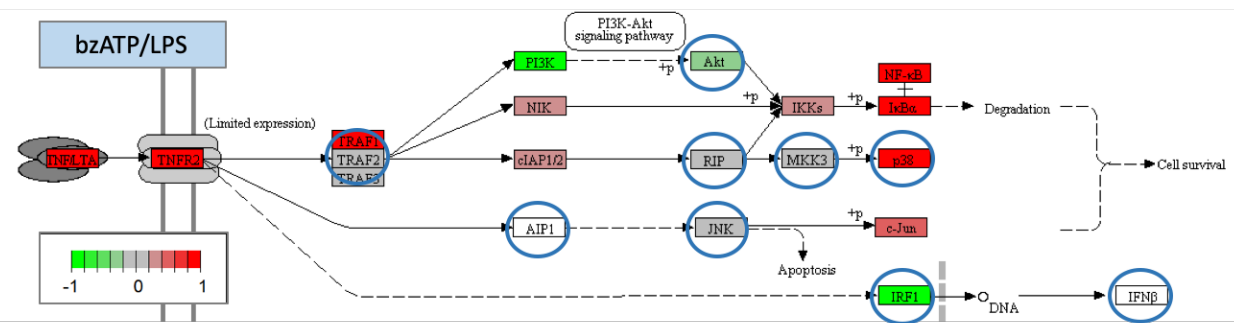

B

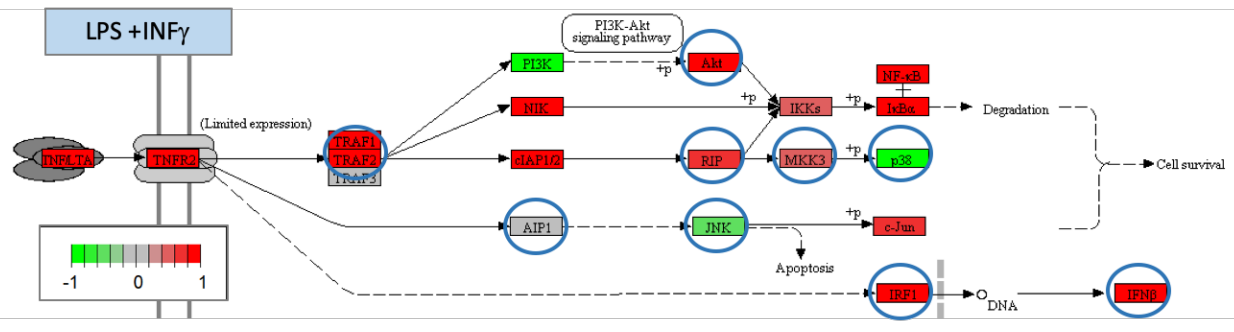

C

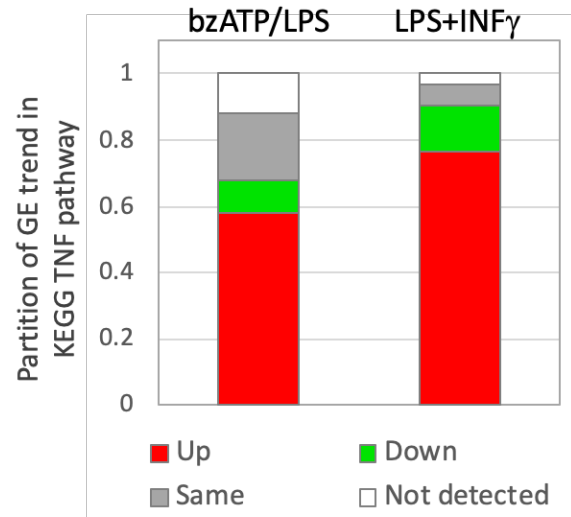

D

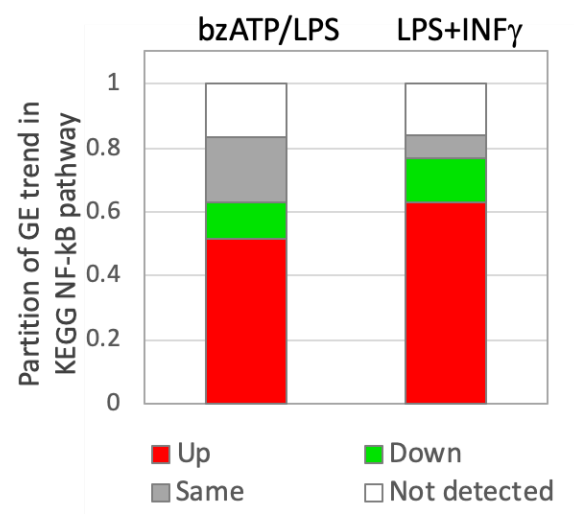

Figure 8. PI3K-Akt pathway from the KEGG pathway map of TNF signaling labelled by DE gene pattern. (A) Microglia culture exposed to bzATP/LPS (8 hrs). (B) Microglia culture exposed to LPS+ IFN $\gamma$ ( 6 hrs). Genes are colored by 4 categories according to by their expression trends. The colors indicate genes that are downregulated (green), upregulated (red), unchanged (gray), and not detected (white) with respect to the expression levels in untreated culture. Genes for which the expression trend differs between $A$ and $B$ are circled (A,B). Partition of all genes listed in each KEGG pathway as summarized for the bzATP/LPS and LPS+IFN $\gamma$ for (C) TNF signaling (Suplementary Fig. S6), and (D) NF-kappa B signaling (Supplementary Fig. S7). Partition to 4 categories (Up, Down, Same and Not detected) matches the colors in A and B. 
A further difference in the signaling cascade between the LPS combined with bzATP or with IFN $\gamma$. Is that apoptosis is induced by activation with LPS+IFN $\gamma$ (see Casp3, Casp7, Supplementary Fig. S6), but these genes are suppressed by bzATP/LPS. The reversed trend was observed for RIP1. RIP1 is a kinase that acts to balance cell death and survival [42]. It is likely that for activation by bzATP/LPS, the signaling cascade shifts towards the NF- $\mathrm{KB}$ branch that initiates MAP kinase signaling as observed for upregulation of p38 (encoded by Mapk11-Mapk14). All other components in this signaling are either unchanged or upregulated. LPS+IFN $\gamma$ down regulates MKK3/6, p38, JNK1/2, and ITCH genes which eventually leads to suppression of AP-1 (activator protein 1), the level of which is fundamental to the execution of the innate immune response.

The overall effect on the entire TNF pathway was tested for the two different stimuli. Fig. 8C shows that the fraction of unchanged genes (=Same, gray) is much smaller for the LPS+IFN $\gamma$ stimulation than for bzATP/LPS. This observation was also generalized to NF-KB signaling (Fig. 8D). We conclude that the nature of the stimulus in cultured microglia differs not only in its kinetics but also in the information in the signal transduction cascade leading to cell response. Among the non-detected genes are transcripts unique to leukocyte recruitment $(\mathrm{Ccc} 15, \mathrm{C} \times 3 \mathrm{cl} 1)$, endothelial cells adhesion (Sele, Vcam1), and vascular effect (Edn1, Vegfc). We conclude that the robust programs of microglial activation with different combinations of stimuli resulted in a rich repertoire of distinct responses that define the underlying biology of the inflammatory routes induced in these cells.

\section{Discussion}

Microglia are characterized by their ability to sense and react to abnormal and disturbing conditions [43]. Because of their crucial importance in maintaining brain homeostasis, the amplitude of the change in their gene expression must be tightly controlled. This study examined the molecular characterization of the response of a microglial neonatal culture to external stimulants. We examined the effect of extracellular ATP (in the form of BzATP) that acts through purinergic receptors on the cell membrane [44]. By means of normalized RNA-seq data we confirmed the exceptional coherence in the levels of gene expression within each experimental condition group. Therefore, all reported results focused on DE genes that satisfied the statistical confidence threshold ( $p$-value FDR $<0.05$ ) and a minimal level of expression. We aimed to distinguish between two alternative models for microglial activation: (i) Activated cells are programmed to converge into a defined transcriptomic profile irrespective of the nature of the stimulus; (ii) The cells are stabilized in several distinct states, each of which displays a unique transcriptional profile according to the physiological scenario. Accordingly, the concentration, duration, and nature of the stimulus (e.g., bzATP, IFN $\gamma$, TNF, growth factors, and their combinations) define the outcome. Our results provide support for the second model in which 
the experimental conditions dictate the detailed cells state as reflected by the underlying signal transduction inflammatory (e.g., TNF and NF-kappa B; Fig. 7).

We showed that bzATP/LPS, induces TNF and IL-6, and their protein products are secreted. While the Tnf gene is highly expressed in naïve cells (ranked 92\% of all genes), there is no indication of its release at the basal state and bzATP, only moderately increased the level of Tnf transcript at $3 \mathrm{hrs}$, by $50 \%$, but increased it considerably ( 65 fold) after the addition of bzATP/LPS. TNF is present in microglia as soluble and membranous versions, each displaying a characteristic binding affinity toward the TNF receptors (Tnfrsf1a and Tnfrsf1b, Supplementary Table S1). The binding of the soluble TNF form is limited to TNF-R1 (a product of the Tnfrsf1a gene) which drives cells to apoptosis and chronic inflammation. In contrast, the membranous form of TNF binds to Tnfrsf1a and Tnfrsf1b. Tnfrsf1b (but not Tnfrsf1a) was strongly upregulated already at $3 \mathrm{hrs}$ by bzATP/LPS ( 4.9 fold; Supplementary Table S4) suggesting additional layers of regulation mediated by TNF. Recently, it was shown that membranous TNF plays a critical role in cell-cell communication through its delivery by extracellular vesicles (EV) [45]. Tnfaip3 is another TNF-related gene that was strongly upregulated, (14.6 fold, Supplementary Table S4) $3 \mathrm{hrs}$ after the addition of bzATP/LPS. Tnfaip3 plays a role in tissue homeostasis through the regulation of NF-KB activation. As shown in Fig. 7 and Supplementary Fig. S8, TNF and NF-kappa B signaling consolidate many of the changes that occurred along the transcriptional waves.

The microglial cell response only become long-lasting following the further addition of LPS which affects hundreds of genes suggesting that priming by bzATP drives microglia to become more sensitive to other stimuli (e.g., LPS). Although 3.1\% of the genes (from 10,835 genes) changed significantly 3 hrs after exposing cells to bzATP, most of them returned to their baseline levels of expression by $8 \mathrm{hrs}$. Thus, we postulate that in vivo, when the stimuli are uncoupled or delayed, activation remains below a critical threshold and the transcriptional wave is likely to fade away. In this study, we show that the microglia culture is able to display a spectrum of cellular responses, making it suitable for testing new reagents and drugs under defined stress conditions (e.g., oxidative stress, hypoxia, ATP concentration, and others).

Different noxious stimuli such as injury, hypoxia, and pathogenic conditions are associated with

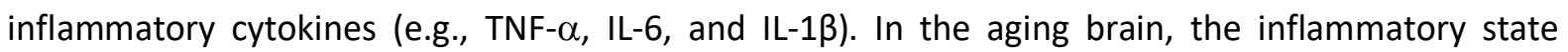
accelerates the progression of neurodegeneration [12]. Consequently, inhibiting microglial activation should be a strategy for slowing down disease progression of the in the aging brain. Recently we showed that ladostigil, a drug with anti-oxidant and anti-inflammatory activity has a beneficial effect on memory decline in the aged rats [46]. Chronic treatment with ladostigil significantly changed the number of activated microglia and their morphology in a region-dependent manner in aging rats [14]. 
The activation cascade in microglia begins with the binding of extracellular molecules to Toll-like receptors [47], thereby resulting in changes in cytokine secretion, cell proliferation, migration, and phagocytosis [48]. In vivo, microglia respond by sensing the local concentrations of molecules, including amyloid $\beta(A \beta)$, nucleic acids, reactive oxygen species (ROS), and ATP. ATP released from dying astrocytes and neurons after injury $[49,50]$ triggers a switch in microglial activity $[51,52]$. Microglia also release ATP through exocytosis [4], thus creating a feedback loop that modulates the activation program. Exposing microglia to bzATP induces a transient wave of DE genes including several that were unexpected and highlights the relevance of genes of the innate immune system for testing drugs in varying conditions [53]. Hence, there was a strong upregulation ( $2.2 \mathrm{fold}, \mathrm{p}$-value 9.3e14) of $\mathrm{Ccl} 2$ (chemokine $\mathrm{C}-\mathrm{C}$ motif ligand 2) that also occurs in the brain of AD disease patients [54]. In mouse models of $A D$, the overexpression of $\mathrm{Ccl} 2$ was shown to cause microglia-induced amyloid $\beta$ oligomerization, worsening of tau pathology, and an increase in IL-6 [55].

The molecular signature of cell activation has been extensively studied in the BV2 microglial cell line [56]. However, the transcriptomes of BV2 and the primary microglial cells are quite different [57, 58]. We show that in bzATP/LPS activated primary microglial culture there is a steady secretion of cytokines and that the induction of cytokines and chemokines is coordinated and increases by several orders of magnitude. Stimulation of neonatal microglia in vitro mimics the complex scenarios occurring in vivo, like exposure to dying neuronal cells (bzATP), viral infection (IFN $\gamma$ ), and pathogens (LPS). IFN $\gamma$ is a strong mediator of a wave of immune-related gene expression [59], so the inflammatory response to viral infection can be simulated by examining its DE profile in primary microglia exposed to LPS+IFN $\gamma$ [37]. A comparison of microglial response to LPS and IFN $\gamma$ (8 hrs in bzATP/LPS and 6 hrs in LPS+IFN $\gamma$ [37] did not substantially change the overall response. In both cellular settings, the overexpressed genes account for the majority of the signal. Nevertheless, the fraction of genes that were unaffected by bzATP/LPS was much higher ( 3 fold) than those affected by LPS+IFN $\gamma$. To see whether this observation is specific to TNF signaling pathway, we tested the inflammationrelated signaling pathway of NF- $\mathrm{\kappa B}$, known as a hub for executing the inflammatory signal in microglia and macrophages [60]. NF-KB is a master regulator linking LPS to the production of IL-10 and IL-6 [61]. We observed that while only $16.5 \%$ of the genes overlap between the TNF and NF-KB signaling pathways, the overall partition of genes according to their expression trend was surprisingly similar between these pathways. Specifically, similar to the overall trends for TNF signaling, for the NF-KB pathway, we also noted many more genes that are marked as unchanged in bzATP/LPS relative to LPS+IFN $\gamma$ treatment (labeled Same, 28 vs. 10 genes).

Even though the studied microglial cell culture cannot capture the complexity of brain injury, aging, or plasticity, it provides a reliable system for mimicking external stimuli. The primary microglial culture 
displayed high responsiveness (i.e., waves of expression with 100s genes) while keeping the variance among repeated experiments exceptionally tight. Despite the lack of communication with other cell types (e.g., neurons, astrocytes, stem cells), the microglial cultures exerted a rich repertoire of responses to external stimuli. We propose adopting the in vitro neonatal primary culture as a model system that senses complex cues from the microenvironment. Such a system is attractive for revealing the underlying mechanism of anti-inflammatory drugs in the ongoing effort to control pathophysiological conditions of the CNS at the cellular level [62].

$\begin{array}{ll}\text { Abbreviations } & \\ \text { AD } & \text { Alzheimer's disease } \\ \text { ATP } & \text { Adenosine tri-phosphate } \\ \text { BSA } & \text { Bovine serum albumin } \\ \text { CNS } & \text { Central nervous system } \\ \text { DE } & \text { Differentially expressed } \\ \text { DMEM } & \text { Dulbecco's modified Eagle medium } \\ \text { FC } & \text { Fold change } \\ \text { FDR } & \text { False discovery rate } \\ \text { GO } & \text { Gene ontology } \\ \text { Hrs } & \text { Hours } \\ \text { IL } & \text { Interleukin } \\ \text { IFNy } & \text { Interferon gamma } \\ \text { LPS } & \text { Lipopolysaccharide } \\ \text { NT } & \text { Not treated } \\ \text { PCA } & \text { Principal component analysis } \\ \text { PD } & \text { Parkinson's disease } \\ \text { RNA-seq } & \text { RNA sequencing analysis } \\ \text { SD } & \text { Standard deviation } \\ \text { TMM } & \text { Trimmed mean of means }\end{array}$

\section{Authors contributions}

All coauthors read and contributed to the final version of the manuscript. K.Z. and E.L. led the data collection, calibration, analyses, statistics and visualization. M.L wrote the manuscript, mentoring and conceptualization; T.E. led the molecular preparation for RNA-seq experiments. F.R. and S.R. led the experimental cell culture and the used protocol; M.W. Conceptualization, writing, review and editing.

\section{Ethical}

All animals related studies were performed according to the National Research Council's guide and approval by the Hebrew University Institutional Committee. The study is reported in accordance with ARRIVE guidelines. Research Ethics ID 15710, Authority for Biological and Biomedical Models, Hebrew University.

\section{Data availability}


RNA-seq data files were deposited in ArrayExpress under the accession E-MTAB-10450.

\section{Funding}

This study was partially supported by the ZC4H2 Associated Rare Disorders (ZARD) Pilot Grant (M.L.).

\section{Acknowledgments}

We would like to thank Linial's lab members for useful comments and fruitful discussion.

\section{References}

1. Amor, S.; Peferoen, L. A.; Vogel, D. Y.; Breur, M.; van der Valk, P.; Baker, D.; van Noort, J. M., Inflammation in neurodegenerative diseases-an update. Immunology 2014, 142, (2), 151-166.

2. Clayton, K. A.; Van Enoo, A. A.; Ikezu, T., Alzheimer's disease: the role of microglia in brain homeostasis and proteopathy. Frontiers in neuroscience 2017, 11, 680.

3. Gao, H.-M.; Hong, J.-S., Why neurodegenerative diseases are progressive: uncontrolled inflammation drives disease progression. Trends in immunology 2008, 29, (8), 357-365.

4. Imura, Y.; Morizawa, Y.; Komatsu, R.; Shibata, K.; Shinozaki, Y.; Kasai, H.; Moriishi, K.; Moriyama, Y.; Koizumi, S., Microglia release ATP by exocytosis. Glia 2013, 61, (8), 1320-30.

5. Sanz, J. M.; Di Virgilio, F., Kinetics and mechanism of ATP-dependent IL-1 beta release from microglial cells. J Immunol 2000, 164, (9), 4893-8.

6. Neher, J. J.; Neniskyte, U.; Brown, G. C., Primary phagocytosis of neurons by inflamed microglia: potential roles in neurodegeneration. Front Pharmacol 2012, 3, 27.

7. Fricker, M.; Vilalta, A.; Tolkovsky, A. M.; Brown, G. C., Caspase inhibitors protect neurons by enabling selective necroptosis of inflamed microglia. J Biol Chem 2013, 288, (13), 9145-52.

8. Hammond, T. R.; Dufort, C.; Dissing-Olesen, L.; Giera, S.; Young, A.; Wysoker, A.; Walker, A. J.; Gergits, F.; Segel, M.; Nemesh, J., Single-cell RNA sequencing of microglia throughout the mouse lifespan and in the injured brain reveals complex cell-state changes. Immunity 2019, 50, (1), 253-271. e6.

9. Wang, W. Y.; Tan, M. S.; Yu, J. T.; Tan, L., Role of pro-inflammatory cytokines released from microglia in Alzheimer's disease. Ann Transl Med 2015, 3, (10), 136.

10. Rodriguez-Gomez, J. A.; Kavanagh, E.; Engskog-Vlachos, P.; Engskog, M. K. R.; Herrera, A. J.; Espinosa-Oliva, A. M.; Joseph, B.; Hajji, N.; Venero, J. L.; Burguillos, M. A., Microglia: Agents of the CNS Pro-Inflammatory Response. Cells 2020, 9, (7).

11. Welser-Alves, J. V.; Milner, R., Microglia are the major source of

Ipha and TGF-beta1 in postnatal glial cultures; regulation by cytokines, lipopolysaccharide, and vitronectin. Neurochem Int 2013, 63, (1), 47-53.

12. Simpson, D. S. A.; Oliver, P. L., ROS Generation in Microglia: Understanding Oxidative Stress and Inflammation in Neurodegenerative Disease. Antioxidants (Basel) 2020, 9, (8).

13. Smith, J. A.; Das, A.; Ray, S. K.; Banik, N. L., Role of pro-inflammatory cytokines released from microglia in neurodegenerative diseases. Brain research bulletin 2012, 87, (1), 10-20.

14. Shoham, S.; Linial, M.; Weinstock, M., Age-Induced Spatial Memory Deficits in Rats Are Correlated with Specific Brain Region Alterations in Microglial Morphology and Gene Expression. J Neuroimmune Pharmacol 2019, 14, (2), 251-262.

15. Shemer, A.; Erny, D.; Jung, S.; Prinz, M., Microglia plasticity during health and disease: an immunological perspective. Trends in immunology 2015, 36, (10), 614-624.

16. Wu, Y.; Dissing-Olesen, L.; MacVicar, B. A.; Stevens, B., Microglia: dynamic mediators of synapse development and plasticity. Trends in immunology 2015, 36, (10), 605-613. 
17. Chen, Z.; Trapp, B. D., Microglia and neuroprotection. Journal of neurochemistry 2016, 136, 10-17.

18. Hickman, S.; Izzy, S.; Sen, P.; Morsett, L.; El Khoury, J., Microglia in neurodegeneration. Nature neuroscience 2018, 21, (10), 1359-1369.

19. Hide, I.; Tanaka, M.; Inoue, A.; Nakajima, K.; Kohsaka, S.; Inoue, K.; Nakata, Y., Extracellular ATP triggers tumor necrosis factor-alpha release from rat microglia. J Neurochem 2000, 75, (3), 965-72.

20. Lambertsen, K. L.; Clausen, B. H.; Babcock, A. A.; Gregersen, R.; Fenger, C.; Nielsen, H. H.; Haugaard, L. S.; Wirenfeldt, M.; Nielsen, M.; Dagnaes-Hansen, F., Microglia protect neurons against ischemia by synthesis of tumor necrosis factor. Journal of Neuroscience 2009, 29, (5), 1319-1330.

21. Cianciulli, A.; Porro, C.; Calvello, R.; Trotta, T.; Lofrumento, D. D.; Panaro, M. A., Microglia Mediated Neuroinflammation: Focus on PI3K Modulation. Biomolecules 2020, 10, (1).

22. Park, J.; Min, J.-S.; Kim, B.; Chae, U.-B.; Yun, J. W.; Choi, M.-S.; Kong, I.-K.; Chang, K.-T.; Lee, D.S., Mitochondrial ROS govern the LPS-induced pro-inflammatory response in microglia cells by regulating MAPK and NF-KB pathways. Neuroscience letters 2015, 584, 191-196.

23. Zhou, L.-t.; Wang, K.-j.; Li, L.; Li, H.; Geng, M., Pinocembrin inhibits lipopolysaccharide-induced inflammatory mediators production in BV2 microglial cells through suppression of $\mathrm{PI3K/Akt/NF-KB} \mathrm{pathway.} \mathrm{European} \mathrm{journal} \mathrm{of} \mathrm{pharmacology} \mathrm{2015,} \mathrm{761,} \mathrm{211-216.}$

24. Akhmetzyanova, E.; Kletenkov, K.; Mukhamedshina, Y.; Rizvanov, A., Different Approaches to Modulation of Microglia Phenotypes After Spinal Cord Injury. Front Syst Neurosci 2019, 13, 37.

25. Reichert, F.; Rotshenker, S., Complement-receptor-3 and scavenger-receptor-AI/II mediated myelin phagocytosis in microglia and macrophages. Neurobiol Dis 2003, 12, (1), 65-72.

26. Gitik, M.; Liraz-Zaltsman, S.; Oldenborg, P. A.; Reichert, F.; Rotshenker, S., Myelin downregulates myelin phagocytosis by microglia and macrophages through interactions between CD47 on myelin and SIRPalpha (signal regulatory protein-alpha) on phagocytes. J Neuroinflammation 2011, 8, 24.

27. Shamash, S.; Reichert, F.; Rotshenker, S., The cytokine network of Wallerian degeneration: tumor necrosis factor-alpha, interleukin-1alpha, and interleukin-1beta. J Neurosci 2002, 22, (8), 3052-60.

28. He, Y.; Taylor, N.; Fourgeaud, L.; Bhattacharya, A., The role of microglial P2X7: modulation of cell death and cytokine release. J Neuroinflammation 2017, 14, (1), 135.

29. Brown, J.; Pirrung, M.; McCue, L. A., FQC Dashboard: integrates FastQC results into a webbased, interactive, and extensible FASTQ quality control tool. Bioinformatics 2017, 33, (19), 3137-3139.

30. Bolger, A. M.; Lohse, M.; Usadel, B., Trimmomatic: a flexible trimmer for Illumina sequence data. Bioinformatics 2014, 30, (15), 2114-2120.

31. Dobin, A.; Davis, C. A.; Schlesinger, F.; Drenkow, J.; Zaleski, C.; Jha, S.; Batut, P.; Chaisson, M.; Gingeras, T. R., STAR: ultrafast universal RNA-seq aligner. Bioinformatics 2013, 29, (1), 15-21.

32. Robinson, M. D.; McCarthy, D. J.; Smyth, G. K., edgeR: a Bioconductor package for differential expression analysis of digital gene expression data. Bioinformatics 2010, 26, (1), 139-40.

33. Eden, E.; Navon, R.; Steinfeld, I.; Lipson, D.; Yakhini, Z., GOrilla: a tool for discovery and visualization of enriched GO terms in ranked gene lists. BMC bioinformatics 2009, 10, (1), 48.

34. Szklarczyk, D.; Franceschini, A.; Wyder, S.; Forslund, K.; Heller, D.; Huerta-Cepas, J.; Simonovic, M.; Roth, A.; Santos, A.; Tsafou, K. P., STRING v10: protein-protein interaction networks, integrated over the tree of life. Nucleic acids research 2014, 43, (D1), D447-D452.

35. Chao, C. C.; Hu, S.; Close, K.; Choi, C. S.; Molitor, T. W.; Novick, W. J.; Peterson, P. K., Cytokine release from microglia: differential inhibition by pentoxifylline and dexamethasone. Journal of Infectious Diseases 1992, 166, (4), 847-853. 
36. Martens, M.; Ammar, A.; Riutta, A.; Waagmeester, A.; Slenter, D. N.; Hanspers, K.; R, A. M.; Digles, D.; Lopes, E. N.; Ehrhart, F.; Dupuis, L. J.; Winckers, L. A.; Coort, S. L.; Willighagen, E. L.; Evelo, C. T.; Pico, A. R.; Kutmon, M., WikiPathways: connecting communities. Nucleic Acids Res 2021, 49, (D1), D613-D621.

37. Pulido-Salgado, M.; Vidal-Taboada, J. M.; Barriga, G. G.; Sola, C.; Saura, J., RNA-Seq transcriptomic profiling of primary murine microglia treated with LPS or LPS + IFNgamma. Sci Rep 2018, 8, (1), 16096.

38. Zhang, J. D.; Wiemann, S., KEGGgraph: a graph approach to KEGG PATHWAY in R and bioconductor. Bioinformatics 2009, 25, (11), 1470-1471.

39. Gao, T.; Jernigan, J.; Raza, S. A.; Dammer, E. B.; Xiao, H.; Seyfried, N. T.; Levey, A. I.; Rangaraju, S., Transcriptional regulation of homeostatic and disease-associated-microglial genes by IRF1, LXRbeta, and CEBPalpha. Glia 2019, 67, (10), 1958-1975.

40. Panda, D.; Gjinaj, E.; Bachu, M.; Squire, E.; Novatt, H.; Ozato, K.; Rabin, R. L., IRF1 Maintains Optimal Constitutive Expression of Antiviral Genes and Regulates the Early Antiviral Response. Front Immunol 2019, 10, 1019.

41. He, Y.; Taylor, N.; Yao, X.; Bhattacharya, A., Mouse primary microglia respond differently to LPS and poly(I:C) in vitro. Sci Rep 2021, 11, (1), 10447.

42. Christofferson, D. E.; Li, Y.; Yuan, J., Control of life-or-death decisions by RIP1 kinase. Annu Rev Physiol 2014, 76, 129-50.

43. Mosher, K. I.; Wyss-Coray, T., Microglial dysfunction in brain aging and Alzheimer's disease. Biochemical pharmacology 2014, 88, (4), 594-604.

44. Illes, P., P2X7 Receptors Amplify CNS Damage in Neurodegenerative Diseases. Int J Mol Sci 2020, 21, (17).

45. Raffaele, S.; Lombardi, M.; Verderio, C.; Fumagalli, M., TNF Production and Release from Microglia via Extracellular Vesicles: Impact on Brain Functions. Cells 2020, 9, (10).

46. Linial, M.; Stern, A.; Weinstock, M., Effect of ladostigil treatment of aging rats on gene expression in four brain areas associated with regulation of memory. Neuropharmacology 2020, 177, 108229.

47. McInturff, J. E.; Modlin, R. L.; Kim, J., The role of toll-like receptors in the pathogenesis and treatment of dermatological disease. J Invest Dermatol 2005, 125, (1), 1-8.

48. Lynch, M. A., The multifaceted profile of activated microglia. Molecular neurobiology 2009, 40, (2), 139-156.

49. Venegas, C.; Heneka, M. T., Danger-associated molecular patterns in Alzheimer's disease. J Leukoc Biol 2017, 101, (1), 87-98.

50. Gulke, E.; Gelderblom, M.; Magnus, T., Danger signals in stroke and their role on microglia activation after ischemia. Ther Adv Neurol Disord 2018, 11, 1756286418774254.

51. York, E. M.; Bernier, L. P.; MacVicar, B. A., Microglial modulation of neuronal activity in the healthy brain. Developmental neurobiology 2018, 78, (6), 593-603.

52. Raouf, R.; Chabot-Doré, A.-J.; Ase, A. R.; Blais, D.; Séguéla, P., Differential regulation of microglial P2X4 and P2X7 ATP receptors following LPS-induced activation. Neuropharmacology 2007, 53, (4), 496-504.

53. Chhor, V.; Le Charpentier, T.; Lebon, S.; Ore, M. V.; Celador, I. L.; Josserand, J.; Degos, V.; Jacotot, E.; Hagberg, H.; Savman, K.; Mallard, C.; Gressens, P.; Fleiss, B., Characterization of phenotype markers and neuronotoxic potential of polarised primary microglia in vitro. Brain Behav Immun 2013, 32, 70-85.

54. Kiyota, T.; Gendelman, H. E.; Weir, R. A.; Higgins, E. E.; Zhang, G.; Jain, M., CCL2 affects $\beta$ amyloidosis and progressive neurocognitive dysfunction in a mouse model of Alzheimer's disease. Neurobiology of aging 2013, 34, (4), 1060-1068.

55. Joly-Amado, A.; Hunter, J.; Quadri, Z.; Zamudio, F.; Rocha-Rangel, P. V.; Chan, D.; Kesarwani, A.; Nash, K.; Lee, D. C.; Morgan, D., CCL2 overexpression in the brain promotes glial activation 
and accelerates tau pathology in a mouse model of tauopathy. Frontiers in Immunology 2020, 11, 997.

56. Butovsky, O.; Jedrychowski, M. P.; Moore, C. S.; Cialic, R.; Lanser, A. J.; Gabriely, G.; Koeglsperger, T.; Dake, B.; Wu, P. M.; Doykan, C. E.; Fanek, Z.; Liu, L.; Chen, Z.; Rothstein, J. D.; Ransohoff, R. M.; Gygi, S. P.; Antel, J. P.; Weiner, H. L., Identification of a unique TGF-betadependent molecular and functional signature in microglia. Nat Neurosci 2014, 17, (1), 13143.

57. Henn, A.; Lund, S.; Hedtjärn, M.; Schrattenholz, A.; Pörzgen, P.; Leist, M., The suitability of BV2 cells as alternative model system for primary microglia cultures or for animal experiments examining brain inflammation. ALTEX: Alternatives to animal experimentation 2009, 26, (2), 83-94.

58. Mendonca, P.; Taka, E.; Bauer, D.; Cobourne-Duval, M.; Soliman, K. F., The attenuating effects of 1, 2, 3, 4, 6 penta-O-galloyl- $\beta$-d-glucose on inflammatory cytokines release from activated BV-2 microglial cells. Journal of neuroimmunology 2017, 305, 9-15.

59. Lively, S.; Schlichter, L. C., Microglia Responses to Pro-inflammatory Stimuli (LPS, IFNgamma+TNFalpha) and Reprogramming by Resolving Cytokines (IL-4, IL-10). Front Cell Neurosci 2018, 12, 215.

60. Hobbs, S.; Reynoso, M.; Geddis, A. V.; Mitrophanov, A. Y.; Matheny, R. W., Jr., LPS-stimulated NF-kappaB p65 dynamic response marks the initiation of TNF expression and transition to IL10 expression in RAW 264.7 macrophages. Physiol Rep 2018, 6, (21), e13914.

61. Heyen, J. R.; Ye, S.-m.; Finck, B. N.; Johnson, R. W., Interleukin (IL)-10 inhibits IL-6 production in microglia by preventing activation of NF-KB. Molecular Brain Research 2000, 77, (1), 138147.

62. Wong, E. T.; Tergaonkar, V., Roles of NF-кB in health and disease: mechanisms and therapeutic potential. Clinical science 2009, 116, (6), 451-465. 


\section{Supplementary Files}

This is a list of supplementary files associated with this preprint. Click to download.

- MGSciRepSupplementalFigS1S7.docx.zip

- SciRepMG14022022SupplementTABLESS1S5.zip 